\title{
Recommendations for the transition of patients with ADHD from child to adult healthcare services: a consensus statement from the UK adult ADHD network
}

\author{
Susan Young ${ }^{1 *}$, Marios Adamou², Philip Asherson ${ }^{3}$, David Coghill ${ }^{4}$, Bill Colley ${ }^{5}$, Gisli Gudjonsson ${ }^{3}$, Chris Hollis ${ }^{6}$, \\ Jane McCarthy ${ }^{3}$, Ulrich Müller ${ }^{7}$, Moli Paul ${ }^{8}$, Mark Pitts ${ }^{9}$ and Muhammad Arif $^{10}$
}

\begin{abstract}
The aim of this consensus statement was to discuss transition of patients with ADHD from child to adult healthcare services, and formulate recommendations to facilitate successful transition. An expert workshop was convened in June 2012 by the UK Adult ADHD Network (UKAAN), attended by a multidisciplinary team of mental health professionals, allied professionals and patients. It was concluded that transitions must be planned through joint meetings involving referring/ receiving services, patients and their families. Negotiation may be required to balance parental desire for continued involvement in their child's care, and the child's growing autonomy. Clear transition protocols can maintain standards of care, detailing relevant timeframes, responsibilities of agencies and preparing contingencies. Transition should be viewed as a process not an event, and should normally occur by the age of 18 , however flexibility is required to accommodate individual needs. Transition is often poorly experienced, and adherence to clear recommendations is necessary to ensure effective transition and prevent drop-out from services.
\end{abstract}

\section{Background}

Transition can be defined as 'the process of change from one stage (state) to another stage (state)'. There are many transitions that an individual makes throughout life, be they through psychosocial stages of development [7] or stages of cognitive development [22], and perhaps the most important transition is from adolescence to adulthood. This is a period of immense significance both in social and psychological terms, as well as in legal terms (i.e. legally becoming an adult at age 18). This is a period where young people are likely to move away from home, either to go to college/university or for job opportunities, start new relationships and assume new roles and responsibilities. This is also a period when many experiment in life, including alcohol and illicit substances. Any disruption during this period is likely to have longer lasting adverse effects on the young person's development,

\footnotetext{
* Correspondence: susan.young1@imperial.ac.uk

${ }^{1}$ Centre for Mental Health, Imperial College London, Broadmoor Hospital,

WLMHT, London, UK

Full list of author information is available at the end of the article
}

realisation of their potential and possibly affect their psychological wellbeing.

Young people with mental health conditions are particularly vulnerable during the transition period, and disruption of care during transition adversely affects the health, wellbeing and potential of this vulnerable group $[15,25]$. Poor transition leads to disruption in continuity of care, disengagement from services and is likely to lead to poor clinical outcomes [25]. Ideally, transition should be a planned, orderly and purposeful process, taking into account developmental and illness specific needs [24]. For the majority of patients transition is poorly planned, poorly executed and poorly experienced $[25,26]$.

\section{ADHD and transition}

Attention deficit hyperactivity disorder (ADHD) is estimated to affect up to five per cent of school-age children and adolescents in the UK [20], with a peak incidence in those aged between six and 12 years [18]. Although ADHD was previously thought of as a childhood condition, meta-analysis of follow-up studies found that $15 \%$ 
of children with ADHD continued to have clinical features that met formal diagnostic criteria at the age of 25 years, and a further $50 \%$ continued to suffer significant impairment into adulthood [8,31]. In addition, comorbid problems such as mood and anxiety disorders and substance misuse persist or develop in adulthood. Other associated problems that may beset adults include difficulties in interpersonal relationships, problems with further education, poor employment opportunities, a propensity for dangerous risk-taking behaviours, and high rates of offending [1,32]. For a review of ADHD in adolescence, see [30]. This constellation of clinical problems and functional impairments emphasises the clear need for mental health services for adolescents and adults with ADHD. Yet data ascertained from 1,636 patients in the UK General Practitioner Research Database, containing data of over three million active patients between the eight year period 1999-2006, reported a great reduction in prescribing patterns during adolescence, in fact hardly any patients were receiving ADHD medication by age 21 [17]. Given the findings of Faraone et al. [8] and Cheung et al. [5], this drop in service utilisation cannot possibly reflect spontaneous remission of ADHD symptoms. Of greater concern is that it occurs at a time when young people need the support of these services the most, as adolescence is a risk period when mental health problems may become more complex and serious disorders emerge.

\section{NICE guidelines}

In 2008, the National Institute for Clinical Health and Excellence (NICE) produced guidance about the recognition and management of ADHD across the life span. They emphasised that ADHD was a life-long condition and provided clear recommendations that care should continue from childhood to adulthood if symptoms persist. They also provided, for the first time in the UK, clear guidance for the development of transition arrangements from child to adult services:

1. Young people with ADHD who are receiving healthcare from child and adolescent mental health services (CAMHS) or paediatric services should normally be transferred to adult mental health services (AMHS) if they continue to have significant symptoms of ADHD or other coexisting conditions that require treatment.

2. Transition should be planned in advance by both the referring service and the receiving service. If needs are severe and/or complex, use of the Care Programme Approach (CPA) should be considered.

3. Patients should be reassessed at school-leaving age to establish the need for continuing treatment into adulthood. If treatment is necessary, arrangements should be made for a smooth transition to adult services, with details of the anticipated treatment and services that the young person will require made clear.

4. Precise timing of the transition arrangements may vary locally, but the transfer should usually be completed by the time the patient is 18 years of age.

5. During the transition to adult services, a formal meeting involving CAMHS and/or the paediatric service and AMHS should be considered, and full information about the adult service should be provided to the patient.

6. For young people aged 16 years and older, the CPA should be used as an aid to transfer between services. The young person, and when appropriate the parent/carer, should be involved in the planning.

7. After transition, a comprehensive patient assessment should be carried out. The assessment should look at personal, educational, occupational and social functioning, and it should evaluate any coexisting conditions, notably drug misuse, personality disorders, emotional problems and learning difficulties.

8. Trusts should ensure that specialist ADHD teams for children, young people and adults jointly develop age-appropriate training programmes for the diagnosis and management of ADHD.

\section{Common practice in the UK}

Although publication of the NICE guidelines has raised awareness of the need for appropriate arrangements for the transfer of young people with ADHD from child to adult healthcare services, AMHS in the UK for ADHD remain patchy. Furthermore, there is significant variation in the ways that services are organized across the UK and it is likely that some of this variation impacts significantly on the effectiveness of transition between adolescent and adult services. Healthcare for children with ADHD is generally provided by either community paediatric services or by CAMHS, or sometimes a combination of the two, that may or may not work in a joinedup manner. The interface between these services and AMHS is often complex with the potential for significant gaps. A survey conducted by Hall et al. [13] reported the absence of joint working between CAMHS and AMHS to be $66 \%$ and $59 \%$ of child and adult services, respectively. Furthermore, all 24 respondents from adult services reported having no official transition policy in place, and $89 \%$ of National Health Service (NHS) Mental Health Trusts reported an absence of dedicated staff to support the transition. Surveys of NHS Trusts have also indicated a nationwide lack of accurate data pertaining to both the number of adults with ADHD within individual trusts, and the number of children/adolescents transitioning to adult services [12]. 
There seems to be a difference in practice between community paediatric and child \& adolescent mental health services. Traditionally, paediatric services have stopped providing care for medical problems early in adolescence, although most now continue to provide care until school-leaving age. In the past most CAMHS generally stopped providing care either at 16 years of age or at school-leaving age (whichever was later). However, there is now a shift towards CAMHS continuing to provide care for patients up to 18 years of age regardless of school status. It has been suggested that AMHS are often too rigid in their age criteria for transition, with reports of refusals to consider transition until the individual reaches the age of 18 [3]. To further complicate issues it is reported anecdotally that whatever the technical cut-off age may be, many paediatric and CAMHS teams continue to see young people well past this age, due to the difficulties in transferring care to adult services [33]. Hence there is a mismatch in the expectation about when care should be passed from adolescent to adult services. Such inconsistencies have been reported to be disruptive and unsettling for patients [28] and this disruption to continuing care is detrimental to overall clinical care from both an individual and service perspective.

\section{Expert workshop}

In order to facilitate further discussion about the transition from child to adult services and develop more explicit and comprehensive recommendations for clinicians, commissioners and policy makers, an expert workshop - "ADHD: Transition from Adolescence to Adult" - was convened in June 2012 in London by the UK Adult ADHD Network (UKAAN) [29]. UKAAN is an organisation founded in 2009 by a group of mental health specialists in response both to the NICE guidelines [19] and to recommendations from the British Association for Psychopharmacology (BAP) [21] that aims to provide support, education, research and training for mental health professionals working with adults with ADHD. The workshop was attended by experts in the field of ADHD, working across services, together with allied professionals and patients. The workshop consisted of a series of presentations summarising the transition process from the perspective of these experts. The workshop first considered the findings of the TRACK study [27] which sought to identify factors that facilitate or impede effective transition more generally within the healthcare system. The National Institute for Health and Care Excellence,[19]) guidelines for transitioning young people with ADHD and the extension to these recommendations proposed by Young et al. [33] were reviewed, and consideration was given to the broader clinical, educational, occupational, and social needs of young people in this age group. There followed an in depth discussion tabling a consensus for detailed recommendations on the provision of transition services for patients with ADHD.

\section{Setting the Context - Findings from the TRACK Study}

The expert workshop commenced with a summary of the findings from the Transitions of Care from CAMHS to AMHS (TRACK) study [27]. The specific aims of the TRACK study were to identify factors that impede or facilitate effective transitions and continuity of care across the mental health services for children and adolescents, covering all disorders and not limited to ADHD, and to make recommendations about the configuration of services.

Healthcare transitions are commonly defined in the literature as a purposeful, planned process that addresses the medical, psychosocial and educational or vocational needs of young people and young adults with chronic physical and medical conditions as they move from child centred to adult orientated healthcare systems. An important context for considering healthcare transition for young people with long-term health conditions is that they typically face several types of transition simultaneously - developmental transitions from childhood to adolescence to adulthood, situational transitions from child services to adult services, and possibly even transitions from relative health to illness or, indeed, sometimes in the other direction. As young people make these transitions they generally take on more responsibility from their parents/carers for all aspects of their healthcare, as well as for other areas of their life. As a consequence it is inevitable that, at the same time, parents/carers face a transition process of their own. Hence the transition from child to adult health services brings service-related challenges associated with an inherent shift from a family-orientated service to an individualorientated service. Whilst the TRACK study focused on those transitioning between child and adult services, not all patients with ADHD will necessarily be in contact with child services at the time of transition. Indeed many of those with ADHD will not have received specific healthcare for their problems during childhood and adolescence - and may be presenting formally for the first time at transition from adolescence to adulthood.

The TRACK project was a multi-site mixed models study, undertaken in six mental health trusts within England. The key findings were:

- Whilst many of the identified transition protocols were based on policy documents, there was evidence of a policy-practice gap, as services differed considerably on their interpretation of both policy and the recommendations for the practical aspects of transition. Although most of the protocols identified the patient as central to the 
transition process, few, if any, specified ways of preparing them for it.

- Optimal transitions were defined as those demonstrating evidence of: information transfer across services; a period of joint working between CAMHS and AMHS; transition planning involving key professionals from both services, patients and/or their parent/carers; and continuity of care.

Transitions were considered sub-optimal if they failed to meet one or more of these criteria. The main findings were that, whilst around $80 \%$ of patients were considered suitable for transition, around one-third were not referred to adult services. Adult services accepted the vast majority of referrals, however of those accepted around $20 \%$ were discharged without being seen and only four per cent experienced optimal transition.

- An organisational analysis showed that CAMHS were seen as being more person-centred, holistic and family orientated, and adult services as more medication focused and crisis orientated. Facilitators for transition included: dedicated transition posts, joint working, early communication and greater involvement of carers. Barriers for transition included variability of eligibility criteria, differing thresholds between AMHS and CAMHS, communication problems between services and lack of confidence amongst adult staff on managing young people in general, and specifically relating to those with neurodevelopmental disorders.

- In a qualitative study of patients', carers' and mental health professionals' experiences of transitions, participants described the importance of transfer planning meetings, joint working between the two services, and good information transfer. Interestingly most young people preferred not having their parents/carers involved with their care at AMHS, and many said that they appreciated the break from this family orientated system because they wanted to be more autonomous. On the other hand parents/carers wanted greater involvement with adult services.

Despite making up a large proportion of the caseloads of CAMHS services, children with ADHD were relatively under-represented within the TRACK sample, making up just under $10 \%$ of the total sample. Only six out of the 15 cases reviewed were transitioned to AMHS and none of these were classified as being 'optimally transitioned'. Overall those young people with neurodevelopmental disorders such as ADHD and autism spectrum disorders, and those with emotional disorders or emerging personality disorders, were more likely to fall through the gaps. Those with serious mental illness, such as psychosis, patients who are receiving pharmacological treatment and with history of inpatient treatment are more likely to be accepted for transition.

\section{Patients' perspectives}

There followed representations from patients on their experience and knowledge of the transition process. The transition period is a time of changing responsibilities, in which the young person is assuming greater autonomy and increased responsibility for his or her own care. Nevertheless, it is important that parents/carers should feel that they are involved in the transition process. This can be challenging because young people do not always want their parents/carers to continue to be involved in their medical care or the management of their condition. However, AMHS need to be willing to work more closely with parents/carers and to recognise that ongoing parental support for the young person is often necessary. This of course can only be achieved with the patient's permission.

It was stressed that transition should start much earlier than is generally recognised. There was a clear call for psychoeducational material for patients and families, as they described wanting to be informed much earlier in the process about their condition and what to expect as they move into adulthood. Specifically they asked for greater detail about their diagnosis and its prognosis, treatment options, involvement in decision making and a specified care plan. In addition, they wanted the opportunity to share with professionals their feelings and their desire to take control of managing their condition and play an active role in their care plan.

It was emphasised that transition needs to be seen as a "process" rather than an "event". Specifically, young people need to know what can be expected of these services, what AMHS offer and what they do not offer. They thought that transition meetings attended by CAMHS and AMHS were a good idea as this would facilitate a smoother transition. The potential value of lifespan clinics in the management of ADHD was highlighted. Patients were mindful however of the support they need in this process due to organisation and planning deficits. Support groups play an important role in the process, providing support for the young person and their family; the latter for the first time have to take a step back with a less proactive role.

The experience described at the workshop strongly resonates with perspectives reported in the Transition Into Adult Mental Health Services (TRAMS) study [28]. This was a qualitative study of the experiences of 10 young people with ADHD, and their parents/carers, in transition from CAMHS to AMHS. Four key themes were identified from the analysis: 
1. Clinician Qualities and Relationship: These were identified to be an integral component of patient satisfaction, with a particular focus on the need for continuity of listening throughout the transition process, non-judgemental support and a practical, solution-focused approach targeting achievable and realistic goals.

2. Responsibility of Care: A number of issues arose regarding the question of who is responsible for providing care and ensuring continuity of care. From the young person's perspective, a conflict can emerge between the greater autonomy expected in AMHS and the difficulties they experience in areas such as planning and organisation, for which they typically rely on parent/carer support. Inevitably for parents/carers, there is a responsibility to balance support and autonomy. Ultimate responsibility for providing a consistent transition lay with clinicians, with the importance of joint meetings between CAMHS and AMHS, alongside patients and their families, as well as an overall clear plan for handover suggested as integral components of a seamless transition.

3. Nature and Severity of Problems: Acceptance by AMHS appeared to be contingent on the nature and severity of the young person's problems. There was a suggestion that transition may go more smoothly for those presenting with more severe problems, such as self-harming behaviours, and higher levels of comorbidity. For those whose problems were well controlled at the time of transition, neither the AMHS nor the young person themselves recognised the need for the on-going support required to sustain this level of health.

4. Expectations of AMHS: The expectations of AMHS from patients and their families were often high, although many of these are unlikely to be met (i.e. assistance with housing). It is essential therefore that CAMHS clinicians do not provide unrealistic expectations of AMHS, with clear information offered prior to transition concerning exactly what can and cannot be offered.

\section{The transition process}

Most AMHS are set up to provide care for patients with serious mental disorders such as psychosis. In the TRACK study, only one in five of the 17 year olds in CAMHS with neurodevelopmental disorders (including ADHD) made a successful transition to adult services [27]. This contrasts with the more successful transition of young people with psychosis and other conditions considered to be serious mental disorders. Moreover, experience suggested that patients with ADHD and comorbid mental health problems, such as depression and suicidal ideation, often have an easier transition to adult services than patients diagnosed with ADHD alone. Nevertheless, one-half of all patients fail to adhere to treatment guidelines [9] and discontinue treatment within 2-3 years of starting pharmacological therapy $[11,34]$. Some common reasons for the discontinuation of treatment are: adverse effects, ineffectiveness/suboptimal response, poor adherence, patient/caregiver decision and symptom relief. Other less common, although important, reasons include a dislike of medication, dosing inconvenience and social stigma [10].

It needs to be emphasised that transition to adult care is a process, rather than an event. For their part, CAMHS should provide timely and accurate information to patients about adult services and develop specific mechanisms to overlap the transitional arrangements with adult services. The transition process can be used to prompt a review of what other organisations may be required in the patient's care. Indeed, this is an opportunity to inform appropriate agencies and organisations, with the patient's consent, of their current circumstances. For this to be achieved, a good working knowledge of the common partner organisations in the area is crucial. These may be statutory and/or local organisations, such as child protection services, the local authority (for assessment for social care), local educational facilities and resources, court diversion services, and youth offending teams. In addition, there are an increasing number of voluntary and third-sector agencies that provide help and support. It is also advisable to involve the patient's general practitioner (GP) because often this is the most stable service provider during the period of transition.

It was proposed that for the transition to adult care to work well, adult psychiatry needs to accept referrals from CAMHS and community paediatric services, and develop a better understanding of their needs. This will involve targeted continuing professional education on ADHD and, in particular, on the transition process. Of concern is that there is no specific provision to ensure ongoing care for ADHD patients who are not accepted by AMHS. There followed discussions regarding the best model for service provision and, in particular, whether adult-focused neurodevelopmental services need to be specifically developed. These already exist in some parts of the UK and were considered useful (see Table 1). To avoid excessive resource implications and an unnecessary division to the process, these are best located as an extension of CAMHS with a set agenda that includes psychoeducation, goal setting, risk reduction, coping strategies, employment and education. Additional consultations can be added on an 'as needed' basis, e.g. with a pharmacist, social worker, psychiatrist, psychologist and/or a member of the youth offending team. 
Table 1 Transition practice in Leicestershire

Leicestershire have developed a robust transition protocol ensuring that all referrals from children's services are carefully reviewed and appropriate action is taken. In Leicestershire, referral acceptance by the adult ADHD service is $100 \%$. Children services are advised to start the referral well in advance of the point of transition on the patient's 18th birthday. They are also advised not to discharge the patient before they have been seen by the adult service. This ensures that the patient is never lost between the two services. In case the patient does not attend the appointment arranged by the adult ADHD clinic, the referring clinician is contacted for further advice and efforts are made to encourage the patient to attend. When dealing with complex cases or difficult to engage patients, a period of joint working has also been found to be useful.

Recognising the vulnerability of patients through this period of transition and the need to ensure continuity of care and stability, a great degree of flexibility is exercised when dealing with this group of patients. Even when the patient is not keen on taking the medication or attending further reviews, they are not discharged from the service. Time is spent with patients providing psychoeducation about the disorder, its course and the consequences of untreated ADHD. During these discussions it is important to acknowledge the patient's views and reservations, for example, stigma about a psychiatric diagnosis label, their wish for autonomy, their view that they can manage their symptoms without the help of medication etc. They are offered a further appointment and are encouraged to take their time before making a final decision. In the large majority of cases, patients decide in favour of continuity of care.

\section{ADHD, education and employment}

We can be fairly confident that key transitions, and in particular, those bridging secondary education and the world of further learning, independent living and work, constitute a period of heightened vulnerability in the life of any young person with ADHD.

However, the absence of a reliable evidence-base to inform generalisations about the employment experience of young people transitioning from school or college to the world of work is complicated by the heterogeneity of the ADHD population in terms of cognitive ability, comorbidity, gender expression, and treatment uptake, as well as geographical variations in the labour market.

Whilst it is fair to suggest that young people with ADHD suit better those occupations that provide novelty, physical activity and immediate feedback, and which are thus non-repetitive, highly structured and lacking in organisational/time-management demands, the prospects for any individual are likely to hinge on a number of other factors which may be either directly or indirectly related to the way that ADHD has manifested itself during their formative years.

Sufficient anecdotal evidence exists to complement studies in USA and Europe to suggest that rates of economic activity and thus income levels are significantly lower for young adults with ADHD, and that such differentials often persist throughout the life span $[4,6,16]$. The treatment effect is poor for occupational outcomes [2], however there is an emerging body of data to support the positive impact of
Table 2 General recommendations for transition of care from children's to adult services

1. Clear transition protocols should be developed jointly by commissioners, CAMHS/paediatric services, AMHS, primary care, and other agencies as relevant to facilitate transition and ensure that standards of care are maintained during the transition period.

2. These protocols should specify timeframes, lines of responsibility, who should be involved, how the young person should be prepared, and what should happen if AMHS are not able to accept the referral.

3. Protocols should allow for flexibility in the age of transition so as to accommodate developmental needs and stages, but there should be explicit referral criteria and service provision. Ideally, transition should occur at a time of clinical stability. Patients should not have to relapse or have worsening mental health in order to continue to be able to access services.

4. Transition protocols should be available to all clinical teams and should include psychoeducational material that provides high-quality, comprehensive, impartial and appropriately written information for both young people and their parents and carers. There is a need for more age-appropriate psychoeducational material for patients at the transition stage. This material should include information about ways that young people can manage their own symptoms and problems, and access advice and support. Information should also be developed in a media format that is readily accessed by young people, e.g. use of phone applications and internet sites.

5. The needs and wishes of parents/carers should be respected and their ongoing involvement with the young person negotiated. Some parallel services that can provide information and support for parents/ carers during the transition period may be required.

6. Efforts should be made to inform and educate allied professionals who may come into contact with young people with ADHD for the first time during the transition period, e.g. forensic medical examiners and those working in the probation services and in correctional units and prisons.

7. Healthcare jurisdictions should be encouraged to use similar care pathways and outcome measures across different patient age groups.

personalised assistance in negotiating the labour market by helping job-seekers with the application and interview process, providing structures in daily living to manage financial obligations and punctuality, and in averting negative tendencies towards substance misuse, social conflict and offending [14, 23]. Likewise, adjustments in the workplace, such as a reframing of organisation demands or support with time-management, may allow workers with deficits in these areas to exploit relative strengths in others and thus provide a net gain to the employer [1]. Programmes to improve the employability of people with ADHD are likely to be most effective when they are sensitive to the current needs and future potential of each individual, multi-modal in delivery, and which address gaps in prior learning that impair recruitment prospects.

\section{Consensus}

Having considered all the available evidence and expert opinion, the expert group deliberated the recommendations that need to be made to achieve a smooth and effective transition from child to adult 
Table 3 Specific recommendations for transition of care from children's to adult services

1. A planned transfer to an adult service should be made if the young person continues to have significant symptoms of ADHD or other COexisting conditions that require treatment

2. Transition should be planned well in advance by both referring and receiving services. Timings of transition may vary but should ordinarily be completed by the age of 18 years. Transition between teams should be a gradual process and should be thought of as a 'process' and not a 'single event'.

3. Patients should be involved in discussion about transition and informed of the outcome of any transition assessment. The transition process should proceed according to need in terms of future medical care (e.g. involvement of general practitioner [GP] services, specialist adult ADHD teams, adult learning disability services, adult physical health teams). Importantly, the GP should be involved throughout the process.

4. Discussion, and where necessary, joint meetings between child and adult services must ensure that the needs of the young person will be appropriately met. It is important to consider the presence of comorbid and/or related problems, which may involve further discussion and collaboration with educational, or occupational and social agencies

5. CAMHS practitioners and paediatricians should foster engagement with AMHS through open discussion and psychoeducation about ADHD, the benefit of evidenced-based psychological and pharmacological treatment where appropriate, and the risks of disengagement. It is important to address concerns about stigma associated with referral to AMHS.

6. For young people aged 16 years or over in CAMHS, a CPA should be used to aid transfer. CPAs are not available in paediatric practice, and so a planned assessment of need with the young person and their parents/carers and a clearly documented plan of action is recommended.

7. Parents/carers need to be prepared and facilitated to aid their child's gradual move towards independence and autonomy (with respect to the management and treatment of their ADHD). The referring and receiving healthcare teams should be mindful of possible parental ADHD and when this is present (or suspected) provide appropriate support.

8. Shared care arrangements between primary and secondary care services for the prescription and monitoring of ADHD medications should be continued into adulthood.

9. Direct psychological treatment should be considered (individual and/or group Cognitive Behavioural Therapy) to support young people during key transitional stages. This should have a skills development focus and target a range of areas including ADHD symptoms, social skills, interpersonal relationship problems (with peers and family), problem solving, self-control, dealing with and expressing feelings. Active learning strategies should be used.

10. Specific protocols need to be developed for young people who are not accepted by AMHS criteria, but whom the referring service strongly believe need ongoing support. Care needs to be taken that these patients are not left without the support they need during this very important transition period.

11. Separate care pathways should be developed for young people who drop out of CAMHS or paediatric services when they are under 18 years of age, and who later re-present in the healthcare system as adults.

12. Separate care pathways should be developed for patients who come to the attention of the healthcare system on account of ADHD for the first time as adults.

13. The referral letter from children's services should provide a comprehensive account of the patient, including: diagnostic summary and formulation; treatment history; rationale and response; side effects,
Table 3 Specific recommendations for transition of care from children's to adult services (Continued)

compliance, abuse and diversion issues, and ongoing treatment needs; any psychiatric and medical comorbidities, their impact on ADHD and treatment; any other ongoing needs - social, financial, accommodation or occupational and an updated risk assessment.

14. The adult service should acknowledge the receipt of the referral. The patient should not be discharged by the children's services until they have been seen by the adult services and their care has formally been taken over by the adult services. This provides a safety net and reduces the likelihood of patients dropping out of the services during the transition period.

15. Following acceptance of the referral, the adult service should allocate a key worker/lead clinician who will coordinate the care needed.

16. When dealing with patients who are anxious about the transfer of care to adult services or those with complex needs, it may be necessary for children's services to joint work with adult services for a few months to facilitate the transfer of care.

services for young people with ADHD, and prevent drop out from services. The earlier recommendations of Young et al. [33] were used as a starting point and framework for discussion. When using these guidelines and recommendations, local situations will need to be considered when designing transition services as there is a considerable variation in service design and delivery between regions. Consensus was reached for general recommendations (Table 2) and specific recommendations (Table 3).

\section{Future research}

In the course of the workshop it became clear that little is known about the outcomes of individuals with ADHD who fall through care gaps, patients who remain under the care of Primary Care services throughout transition into adulthood, interventions that might improve the process, and patient and carer experiences. Thus recommendations were also made for future research into areas relevant to the transition of patients with ADHD from child to adult services, including:

1. The reasons why patients stop taking medication during the transition period.

2. The reasons why patients lose contact with their ADHD healthcare system during the transition period, including why adolescents chose to discontinue treatment and how to address issues of decreased efficacy of medication over time.

3. Pharmacological strategies to identify patients, before their transition to adult services, who are at risk of developing comorbid psychiatric difficulties, with a view to preventing the development of these comorbid problems.

4. The effectiveness of non-pharmacological interventions in this age group. 
5. Females with ADHD are underrepresented in the research literature, and their position through the transition process demands more attention.

\section{Conclusions}

Transition is a process, not an event, and normally occurs by the age of 18 . However, flexibility is required to accommodate individual needs. Unfortunately, transition is often poorly planned, executed and experienced. Adherence to clear recommendations is necessary to ensure effective transition and prevent drop-out from services.

\section{Acknowledgements}

The authors wish to acknowledge the following individuals who attended the consensus meeting: Blanca Bolea, Helen Crimlisk, Helen Duncan, Susan Dunn Morua, Val Harpin, Claude Jousselin, Geoff Kewley, Angela Koni Moore, Clodagh Murphy, Mavis Nyakunengwa, Shorayi Nyamupanda, Fintan O'Regan, Sri Perecherla, Prem Shah. In addition we acknowledge the assistance of Ben Greer for the preparation of the manuscript.

\section{Funding}

No funding was obtained for this study.

\section{Availability of data and materials}

Not Applicable - not datasets were generated or analysed in the course of the expert workshop.

\section{Authors' contributions}

All authors contributed to the planning and scientific input of this consensus statement. Early drafts of the manuscript were completed by SY, DC and MAr. All authors read and approved the final manuscript.

\section{Authors' information}

Susan Young obtained a doctorate in clinical psychology from University College London in 1999 and a PhD in psychology from King's College London in 1999. She is a Senior Lecturer in Forensic Clinical Psychology at Imperial College London, and Director of Forensic Research \& Development at West London Mental Health NHS Trust. Previously, she was employed as a Clinical Neuropsychologist at the Maudsley Hospital, where she set up and developed the neuropsychology service at the first adult ADHD service in the United Kingdom, and has extensive clinical experience in the assessment and treatment of youths and adults with ADHD and in the assessment and treatment of offenders with mental illness and/or mental disorder. Her main research interests are in the areas of neurodevelopmental disorder, ADHD, and forensic psychology.

Marios Adamou is a Consultant Psychiatrist in Neurodevelopmental Psychiatry (ADHD and ASD) at South West Yorkshire Partnership NHS Foundation Trust, and Professor at Huddersfield University, UK. His medical training was completed at the Aristotle University, Greece, and his psychiatric training at Guy's, King's, and St Thomas's Hospitals, London. He holds numerous positions including fellow of the Higher Education Academy (FHEA), the Royal Society of Arts (FRSA), the Royal Society of Public Health (FRSPH) and the Society of Biology (FSB). Philip Asherson is Professor of Molecular Psychiatry at the MRC Social, Genetic and Developmental Psychiatry centre at the Institute of Psychiatry, Psychology and Neuroscience, King's College London. He is also a Consultant Psychiatrist at the Maudsley Hospital. He earned his medical degree from The Royal London Hospital and his doctoral degree from the University of Wales. His current research interests focus upon clinical, quantitative genetic and molecular genetic studies of ADHD across the lifespan, investigation of mood instability and emotional regulation in ADHD, and treatment of ADHD in young offenders.

David Coghill leads the Developmental Research Group at the University of Dundee. His group's research interests are focused on ADHD and other developmental disorders and include studies into the neuropsychology of ADHD and conduct disorder, neuropsychopharmacoloogy, genetics, pharmacogenetics, clinical trials, quality of life, and translational studies investigating the best ways to integrate scientific knowledge into clinical practice.
Bill Colley is a writer, teacher trainer and educational consultant who spent much of his career in the independent sector before becoming the headmaster of a residential special school. He is currently working in local government as a Service Manager with responsibility for additional support needs and contributes at a local level to an ADHD support group, the NHS Autism Assessment Pathway, and to the National Autism Strategy Reference Group (Scotland). He is an advisor to Mindroom, a Scottish charity for people with learning difficulties and has a particular interest in improving educational outcomes for children who experience developmental disorders, and in supporting their families via multiprofessional collaboration. His latest task is to establish a new provision, and an authority-wide service, for children who have disengaged from mainstream education.

Gisli Gudjonsson CBE, obtained a PhD from the University of Surrey, England, in 1981. He is Emeritus Professor of Forensic Psychology at the Institute of Psychiatry, Psychology \& Neuroscience King's College London. His main research interests are in the areas of forensic psychology, including police interrogation, false confessions, and motivation for offending. In 2009 the British Psychological Society granted him a Lifetime Achievement Award for his exceptional and sustained contribution to the practice of psychology. He was appointed a Commander of the Order of the British Empire (CBE) in the Queen's Birthday 2011 Honours List for services to clinical psychology. Chris Hollis is Professor of Child \& Adolescent Psychiatry at the University of Nottingham and Consultant in Developmental Neuropsychiatry with Nottinghamshire Healthcare NHS Trust. Before moving to Nottingham, Chris trained in psychiatry at the Maudsley Hospital and Institute of Psychiatry, where he completed his PhD. His research interests include ADHD, Tourette syndrome, early onset schizophrenia and the development and implementation of digital technologies to enhance assessment and monitoring of mental health disorders. Over the last 5 years Chris has a research income of over $£ 2.5$ million. Chris was a member of the NICE Guideline Development Group (GDG) for ADHD (2005-8), NICE ADHD Quality Standard Advisory Committee (2012-13) and chaired the NICE GDG for schizophrenia and psychosis in children and young people (2011-13). Jane McCarthy is a Consultant Psychiatrist in Intellectual Disability with the East London NHS Foundation Trust and is a Visiting Senior Lecturer at King's College London, England. She has worked for over 20 years as a senior psychiatrist in specialist services for adolescents and adults with intellectual disabilities and autism. Dr. McCarthy's key research interests include the outcomes of psychiatric disorders in people with neurodevelopmental disorders, and in 2012 she was elected Chair of the International Association for Scientific Study of Intellectual and Developmental Disabilities (IASSIDD) Challenging Behavior \& Mental Health Special Interest Research Group, which is the largest global research network in this field of research. She is also Vice Chair of the Psychiatry of Intellectual Disability Faculty, Royal College of Psychiatrists, UK.

Ulrich Müller is a Senior Lecturer in the Department of Psychiatry, University of Cambridge, UK, and Honorary Consultant Psychiatrist with Cambridge University Hospitals NHS Foundation Trust and Cambridgeshire \& Peterborough Foundation NHS Trust (CPFT). Previously he was trained in psychiatry, psychotherapy, neurological rehabilitation and cognitive neuroscience in Germany. His research focuses on adult ADHD and cognitive enhancing medication, with recent studies clarifying how drugs such as atomoxetine and methylphenidate modulate cognitive functions and related brain processes. Moli Paul is a principal teaching fellow at the University of Warwick and an honorary consultant child and adolescent psychiatrist with Coventry and Warwickshire Partnership Trust, UK. Her main research interests are health care decision-making by children, young people and their families; transitions between adolescent and adult health services; and empirical research on consent and rights in biomedical ethics and health care law. Mark Pitts is the Clinical Nurse Specialist at the Adult ADHD Service, an outpatient clinic based at the Maudsley Hospital, and one of a number of national specialist services offered by the South London \& Maudsley NHS Foundation Trust. He has degrees in Psychology and Learning Disability Nursing, and worked for five years in a national low-secure service for people with mild/borderline learning disability at the Bethlem Royal Hospital prior to taking up his current position in 2005. His clinical interest are in the assessment of ADHHD in adults, pharmacological treatment and follow-up, and the development of adult ADHD services.

Muhammad Arif trained in Psychiatry in Pakistan, where he gained experience in the assessment and treatment of children with ADHD. He is currently acting Consultant Psychiatrist working in Leicester, UK, where he 
formed the special interest Adult ADHD clinic in 2002. In January 2009 he was responsible for the commission of the Adult ADHD service in Leicester. Other interest include Trauma Psychiatry, CBT and EMDR.

\section{Competing interests}

SY has received honoraria for consultancy, sponsorship for attendance at scientific meetings, educational talks and /or research awards from Janssen, Lilly, Novartis, HB Pharma, Flynn Pharma and/or Shire. King's College London departmental research support account for PA received honoraria for consultancy, educational talks and/or research awards from Shire, Lilly, Novartis, Vifor Pharma, GW Pharma, Janssen and/or QbTech. DC has received honoraria for consultancy, educational talks and/or research awards from Novartis, Sandoz, Vifor, Shire, Lilly and/or Janssen. GG has received consultancy fees, speaker fees and/or travel honoraria from Lilly, Janssen and Shire. The University of Nottingham has received research funding for $\mathrm{CH}$ from Shire for non-pharmacologicial ADHD research. UM has received honoraria for consultancy, educational talks and sponsorship for attendance at scientific meetings from Lilly, Flynn Pharma/Medice, Janssen and/or Shire. MPi has received honoraria for consultancy and educational talks from Shire. MA has received honoraria for consultancy, educational talks and sponsorship for attendance at scientific meetings from Lilly, Janssen Cilag, Otsuka, Servier, Flynn-Pharma. Other authors have no competing interests to declare.

\section{Consent for publication}

Not applicable.

\section{Ethics and consent to participate}

Not applicable.

\section{Author details}

Centre for Mental Health, Imperial College London, Broadmoor Hospital, WLMHT, London, UK. ${ }^{2}$ School of Human and Health Sciences, University of Huddersfield, Queensgate, Huddersfield, UK. ${ }^{3}$ Institute of Psychiatry, Psychology \& Neuroscience (loPPN), King's College London (KCL), London, UK. ${ }^{4}$ University of Dundee, Dundee, UK. ${ }^{5}$ Education and Children's Services, Perth, UK. ${ }^{6}$ Division of Psychiatry and Applied Psychology, Institute of Mental Health, University of Nottingham, Nottingham, UK. ${ }^{7}$ Cambridge University, Cambridge, UK. ${ }^{8}$ Warwick Medical School, Coventry, UK. ${ }^{9}$ South London and Maudsley NHS Foundation Trust, London, UK. ${ }^{10}$ Leicester Adult ADHD Service, Leicester, UK.

\section{Received: 29 January 2016 Accepted: 18 August 2016} Published online: 26 August 2016

\section{References}

1. Adamou M, Arif M, Asherson P, Aw TC, Bolea B, Coghill D, Young S. Occupational issues of adults with ADHD. BMC psychiatry. 2013;13(1):59.

2. Arnold LE, Hodgkins P, Caci H, Kahle J, Young S. Effect of treatment modality on long-term outcomes in attention-deficit/hyperactivity disorder: a systematic review. PloS one. 2015;10(2):e0116407.

3. Belling R, McLaren S, Paul M, Ford T, Kramer T, Weaver T, Singh SP. The effect of organisational resources and eligibility issues on transition from child and adolescent to adult mental health services. J Health Serv Res Policy. 2014;19(3):169-76.

4. Biederman J, Faraone SV. The effects of attention-deficit/ hyperactivitydisorder on employment and household income. Medscape General Medicine. 2006;8(3):12.

5. Cheung CH, Rijdijk F, McLoughlin G, Faraone SV, Asherson P, Kuntsi J. Childhood predictors of adolescent and young adult outcome in ADHD. J Psychiatr Res. 2015;62:92-100.

6. Daley $\mathrm{D}$, Jacobsen $\mathrm{RH}$, Lange $\mathrm{AM}$, Sorensen A, Walldorf J. Private and social costs of ADHD: Cost analysis, The Rockwool Foundation Research Unit Study Paper (Book 69). Copenhagen, Denmark: University Press of Southern Denmark; 2014

7. Erikson EH. Childhood and Society. 2nd ed. New York: Norton; 1963.

8. Faraone SV, Biederman J, Mick E. The age-dependent decline of attention deficit hyperactivity disorder: a meta-analysis of follow-up studies. Psychol Med. 2006;36(2):159-65.

9. Ferrin M, Taylor E. Child and caregiver issues in the treatment of attention deficit-hyperactivity disorder: education, adherence and treatment choice. Future Neurol. 2011;6(3):399-413.
10. Gajria K, Lu M, Sikirica V, Greven P, Zhong Y, Qin P, Xie J. Adherence, persistence, and medication discontinuation in patients with attentiondeficit/hyperactivity disorder-a systematic literature review. Neuropsychiatr Dis Treat. 2014;10:1543.

11. Garbe E, Mikolajczyk RT, Banaschewski T, Petermann U, Petermann F, Kraut AA, Langner I. Drug treatment patterns of attention-deficit/ hyperactivity disorder in children and adolescents in Germany: results from a large population-based cohort study. J Child Adolesc Psychopharmacol. 2012;22(6):452-8.

12. Hall CL, Newell K, Taylor J, Sayal K, Hollis C. Services for young people with attention deficit/hyperactivity disorder transitioning from child to adult mental health services: A national survey of mental health trusts in England. J Psychopharmacol. 2015;29(1):39-42.

13. Hall CL, Newell K, Taylor J, Sayal K, Swift KD, Hollis C. 'Mind the gapmapping services for young people with ADHD transitioning from child to adult mental health services. BMC psychiatry. 2013;13(1):186.

14. Kubik J. Efficacy of ADHD coaching for adults with ADHD. J Atten Disord. 2010;13(5):442-53.

15. Lamb, C., Hall, D., Kelvin, R., Van Beinum, M. (2008). Working at the CAMHS/ Adult Interface: Good practice guidance for the provision of psychiatric services to adolescents/young adults. Retrieved from http://www.rcpsych.ac. uk/pdf/Transition_2008.pdf

16. Le HH, Hodgkins P, Postma MJ, Kahle J, Sikirica V, Setyawan J, Doshi JA Economic impact of childhood/adolescent ADHD in a European setting: the Netherlands as a reference case. Eur Child Adolesc Psychiatry. 2014; 23(7):587-98.

17. McCarthy S, Asherson P, Coghill D, Hollis C, Murray M, Potts L, Wong IC. Attention-deficit hyperactivity disorder: treatment discontinuation in adolescents and young adults. Br J Psychiatry. 2009;194(3):273-7.

18. McCarthy S, Wilton L, Murray ML, Hodgkins P, Asherson P, Wong IC. The epidemiology of pharmacologically treated attention deficit hyperactivity disorder (ADHD) in children, adolescents and adults in UK primary care. BMC Pediatr. 2012;12(1):78.

19. National Institute for Health and Care Excellence. Attention deficit hyperactivity disorder: Diagnosis and management of ADHD in children, young people and adults, NICE clinical guidance 72. London: NICE; 2008.

20. National Institute for Health and Care Excellence. Methylphenidate, atomoxetine and dexamfetamine for the treatment of attention deficit hyperactivity disorder (ADHD) in children and adolescents, Technology Appraisal Guidance [TA98]. London: NICE; 2006.

21. Nutt DJ, Fone K, Asherson P, Bramble D, Hill P, Matthews K, Young S. Evidence-based guidelines for management of attention-deficit/ hyperactivity disorder in adolescents in transition to adult services and in adults: recommendations from the British Association for Psychopharmacology. J Psychopharmacol. 2007;21(1):10-41.

22. Piaget J. Piaget's theory. Berlin: Springer; 1976

23. Ratey N. Life coaching for adult ADHD. In: Goldstein S, Ellison AT, editors. Clinician's guide to adult ADHD: Assessment and Intervention. London: Academic; 2002. p. 261-79.

24. Royal College of Paediatrics and Child Health. (2003). Bridging the gaps: Health care for adolescents. The Royal College of Psychiatrists. Council report: CR1 14.

25. Singh SP. Transition of care from child to adult mental health services: the great divide. Curr Opin Psychiatry. 2009;22(4):386-90.

26. Singh SP, Evans N, Sireling L, Stuart H. Mind the gap: the interface between child and adult mental health services. Psychiatrist. 2005;29(8):292-4.

27. Singh SP, Paul M, Ford T, Kramer T, Weaver T, McLaren S, White S. Process, outcome and experience of transition from child to adult mental healthcare: multiperspective study. Br J Psychiatry. 2010;197(4):305-12.

28. Swift KD, Hall CL, Marimuttu V, Redstone L, Sayal K, Hollis C. Transition to adult mental health services for young people with Attention Deficit/ Hyperactivity Disorder (ADHD): a qualitative analysis of their experiences. BMC psychiatry. 2013;13(1):74.

29. UK Adult ADHD Network. (2012). Transition of ADHD from adolescence to adulthood. Retrieved from: //www.ukaan.org/ADHD-Adolescence-Adulthood/. Accessed 23 Aug 2016

30. Young S. ADHD in Adolescence. 2014; Retrieved from http://www. psychology-services.uk.com/adhd-in-adolescence.htm. Accessed 23 Aug 2016.

31. Young S, Gudjonsson G. Growing out of Attention-Deficit/Hyperactivity Disorder: the relationship between functioning and symptoms. J Atten Disord. 2008;12(2):162-9. 
32. Young S, Moss D, Sedgwick O, Fridman M, Hodgkins P. A meta-analysis of the prevalence of attention deficit hyperactivity disorder in incarcerated populations. Psychol Med. 2015;45(2):247-58.

33. Young S, Murphy CM, Coghill D. Avoiding the 'twilight zone': recommendations for the transition of services from adolescence to adulthood for young people with ADHD. BMC psychiatry. 2011;11(1):174.

34. Zetterqvist J, Asherson P, Halldner L, Långström N, Larsson H. Stimulant and non-stimulant attention deficit/hyperactivity disorder drug use: total population study of trends and discontinuation patterns 2006-2009. Acta Psychiatr Scand. 2013;128(1):70-7.

Submit your next manuscript to BioMed Central and we will help you at every step:

- We accept pre-submission inquiries

- Our selector tool helps you to find the most relevant journal

- We provide round the clock customer support

- Convenient online submission

- Thorough peer review

- Inclusion in PubMed and all major indexing services

- Maximum visibility for your research

Submit your manuscript at www.biomedcentral.com/submit
Biomed Central 